First publ. in: Journal of Physical Chemistry / B, 101 (1997), 49, pp. 10162-10165

\title{
Atomic Force Microscope Based Kelvin Probe Measurements: Application to an Electrochemical Reaction
}

\author{
M. Böhmisch,* F. Burmeister, A. Rettenberger, J. Zimmermann, J. Boneberg, and P. Leiderer \\ Universität Konstanz, Fakuliär für Physik, D-78434 Konstanz, Germany
}

\begin{abstract}
An atomic force microscope (AFM) was utilized as a Kelvin probe to determine work functions of several metals and semiconductors quantitatively. Most of the experimental data show excellent agreement with published values measured by photoemission. Variations in work functions as low as $5 \mathrm{mV}$ could be detected with a typical lateral resolution of $20 \mathrm{~nm}$. This method allowed us to analyze and explain the energetics of an electrochemical reaction on the surface of $\mathrm{WSe}_{2}$, which could be in situ induced and controlled by an externally applied voltage between AFM tip and sample. Thus it could be exploited for etching nanostructures.
\end{abstract}

\section{Lntroduction}

In recent years there has been a rapid progress in scanning probe methods. Today these techniques are not only used for visualizing surfaces but also for their manipulation, both on a nanometer or even atomic scale. ${ }^{1,2}$ Moreover, much effor has been directed toward a mapping of surface properties in order to obtain material contrast, which is attributed to differences in, for instance, adhesion, ${ }^{3.4}$ friction, ${ }^{5.6}$ conductivity, ${ }^{7.8}$ work function, ${ }^{9-19}$ optical absorption, or emission. ${ }^{20}$ Among these, the determination of work function differences (which is the contact potential) seems to be less common in the literature and mainly deals with dopant profiling in semiconductor devices. ${ }^{14-19}$ In these experiments, the well-known Kelvin probe technique ${ }^{2122}$ has been adopted to atomic force microscopy. ${ }^{12-19}$ By com. bining these methods, the topography and the contact potential between the probing tip and the sample can be measured simultancously, provided that tip and sample are electrically connected and conductive. Here, in contrast to the displacement current based conventional Kelvin probe technique, the elecirosiatic force between tip and sample is used for sensing the contact potential.

Up to now, this combination of AFM and Kelvin probe has only been utilized for analyzing lateral structures such as dopant profiles by comparing the contact potentials of the sample's surface and the tip at different sites of the surface. Therefore it has not been possible to obtain absolute and quantitative values of the sample's work function. This rescriction could be circumvented as follows: In the case of clean surfaces, the contact potential between two conducting materials is generally the difference in their work functions. Thus, after calibrating the probing lip against a substrate with a well-known work function as a reference, the absolute value of a sample's work function can be determined.

In the current investigation, this approach was used to examine work functions of metals and semiconductors quantitatively. Moreover, we exploited this possibility to interpret our experimental findings of a local electrochemical reaction on semiconducting $\mathrm{WSe}_{2}$ under ambient conditions. ${ }^{23}$ This corrosion reaction could be induced and controlled by the height of a de voltage externally applied between the AFM tip and the sample.

* Corresponding author. Fax, +49 7531 882627; e-mail, mathias.boehnisch@upi-konstanz.de.

Abstract published in Advance ACS Abstracts. November 15. 1997.

\section{Experimental Section}

All experiments were carried out under ambient conditions using a home-built atomic force microscope (AFM), ${ }^{24}$ It was eitber operated in "contact mode" for the purpose of mapping the surface and determining the conditions necessary for its modification ("etching") or in the combination of "noncontact mode" and "Kelvin probe" (furher referred to as "Kelvin mode") in order to measure topography and contact potential simultaneously. In this setup, the AFM tip, i.e., the cantilever, is mechanically driven near its resonance frequency $f_{\text {res. }}$. When it approaches the surface the oscillation will be damped due to attractive van der Waals forces. The resulcing shifts in vibrational phase and amplitude can both be used for maintaining a feedback loop. We have usually used the phase shift to keep a constant distance between the AFM tip and the sample, thus yielding the topography of the surface.

Additionally, if tip and sample are electrically connected and sufficiently conductive, they will form a capacitance $C$ charged by the contact potential between the two materials. In the present work a small electrical ac field $V_{\text {ac }}$ (typically in the range of $100-700 \mathrm{mV}$ ) with frequency $f_{\text {cp }}=2 \mathrm{kHz}, f_{\text {res }}$, and a variable $\mathrm{dc}$ vollage $V_{\mathrm{dc}}$ were applied between ip and sample for measuring the electrostatic force caused by their contact potential. As the total electrostatic force $F$ is proportional to the square of all the potential differences involved, one gets

$$
\begin{aligned}
& F \approx- \mathrm{d} C / \mathrm{d} z\left[\left(V_{\mathrm{cpd}}+V_{\mathrm{dc}}\right)^{2}+2\left(V_{\mathrm{ac}} / 2\right)^{2}+\right. \\
&\left.\left(V_{\mathrm{cpd}}+V_{\mathrm{dc}}\right) V_{\mathrm{ac}} \sin \left(\omega_{\mathrm{cpd}} t\right)+\left(V_{\mathrm{ac}} / 2\right)^{2} \cos \left(2 \omega_{\mathrm{cpd}} t\right)\right]
\end{aligned}
$$

Obviously, the amplitude of the $w_{\text {cpd }}$ dependent component $F\left(w_{\text {cpd }}\right)$ is proportional to $\left(V_{\mathrm{dc}}+V_{\mathrm{cpd} d}\right)$. In the Kelvin mode, this component was measured by lock-in technique. Then a second feedback loop varied the external dc voltage $V_{\text {dc }}$ such that $F\left(w_{\text {cod }}\right)$ was compensated to zero similiar to the conventional Kelvin probe technique. Thus the acnually required $V_{\mathrm{dc}}$ for compensation is a direct measurement of the contact potential difference. This selup provides minimum cross-talk between contact potential and topographic data. Its performance will be discussed later. All data presented here are raw and unfiltered except an occasional first-order plane subtraction in topography.

Standard cantilevers (of typical spring constant $k=0.1 \mathrm{~N} / \mathrm{m}$ and resonance frequency $f_{\mathrm{res}}=(3 \mathrm{kH} x)^{25}$ originally manufactured and designed for "contact mode" operation were used in both operating modes. They consisted of highly n-doped Si which was sufficiently conductive $(0.01 \mathrm{~cm})$. Measurements 


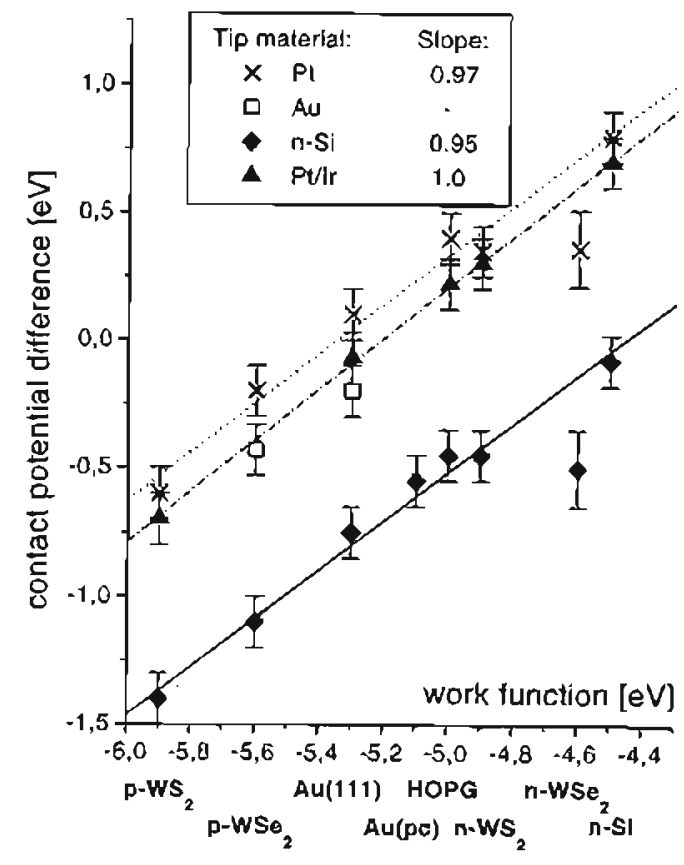

Figure 1. Dependence of the contact potentials measured with the AFM Kelvin mode on the work functions of different substrates (taken from literature) and (ip materials. The lines represent linear regressions on the daca (leaving out the data points for $n-W_{S} e_{2}$ ). The corresponding slopes obtained in this way are given in the graph.

TABLE 1: Comparison between the Experimentally Determined Work Functions-Referred to the Vacunm Level-and Data from Literature for Different Tip Materials

\begin{tabular}{lll}
\hline \multicolumn{1}{c}{ ip } & $\Phi_{\text {tip }}$ exptl $(\mathrm{eV})$ & \multicolumn{1}{c}{$\Phi_{\text {tip }}$ lit. (eV) [reference] } \\
\hline $\mathrm{n}-\mathrm{Si}$ & $-4.5 \pm 0.1$ & $-4.4,-4.5[26-28]$ \\
$\mathrm{Pr}$ & $-5.4 \pm 0.1$ & $-5.6(\mathrm{pc}),-5.7(111)[26,28]$ \\
$\mathrm{P} / \mathrm{I} \mathrm{cs}$ & $-5.3 \pm 0.1$ & $-5.27(\mathrm{pc}),-5.6(111)[26,28]$ \\
$\mathrm{Au}$ & $-5.1 \pm 0.15$ & $-5.1(\mathrm{pc})[26,28]$
\end{tabular}

were performed with bare as well as with metal-coated cantilevers, the first naturally covered by an oxide layer. In noncontact mode, the working distance between AFM tip and the surface was controlled to about $10-15 \mathrm{~nm}$ with a vibrating amplitude in the range of $0.5-0.8 \mathrm{~nm}$. In contact mode, the applied forces were generally in the order of $1 \mathrm{nN}$.

\section{Results and Discussion}

In a first set of experiments, the capability of the "Kelvin mode" for measuring work functions of several materials is demonstrated quantitatively.

As the contact potential difference (CPD) should strictly depend on the materials involved, we determined the CPD of several tip and sample combinations. Ideally, one would expect that the values obtained for different cantilever materials show a linear dependence on the samples work functions-literature data mostly obtained by photoemission ${ }^{26-29}$-with a slope of 1. In fact, according to Figure 1, our data follows this prediction well within the experimental errors. The lines represent linear regressions on the experimental data sets. Of course, a larger set of measurements with different CPD would be of interest. But we aimed at calibrating the cantilevers and were thus restricted to a few materials with surfaces well-known for their chemical stability and inertness under ambient conditions. The values, obtained in this way, of the cantilevers' work functions are summarized in Table 1 togetber with data taken from literature. Furthernore we observed that the calibration was valid as long as the tips remained in their original configuration. For example, upon loss of the tip's coating by wear or changing its surface by lubrication, one could detect a change of the actually measured CPD caused by a variation of the tip's work function.

Up to now, we always had to exchange either the cantilevers or the samples for determining the contact potential of a certain material combination. For demonstrating the lateral resolution and the reliability of our method, one would like to have an in situ contrast of at least two different materials above taken as references. Consequently a $20 \mathrm{~nm}$ gold film was evaporated on HOPG (highly ordered pyrolytic graphite), which was freshly cleaved before mounting it in the evaporation chamber. During the evaporation process the graphite surface was partly shielded using an evaporation mask as illustrated in Figure 2a. 'This mask consisted of circular boles baving $3.6 \mu \mathrm{m}$ diameter, hexagonally arranged with a spacing of $10 \mu \mathrm{m}$. It was fabricated with the LIGA technique. ${ }^{30}$ Figure $2 b$ represents the topography of the so-formed transition areas between the coated and uncoated graphite. Obviously, the mask was not perfectly aligned to the HOPG surface, which led to smearing out of the gold pattern. Figure 3 shows a transition area between the evaporated gold and the HOPG substrate in more detail. Topography (Figure 3a) reveals the Volmer Weber growth mode, which is typical and well-known for the deposition of gold on graphite. ${ }^{31}$ The contact potentials (Figure $3 b$ ) vary exactly with the corresponding materials, especially visible on the isolated gold islands. Moreover, according to literature data (see Figure 1), the gold exhibits a work function about $90 \mathrm{meV}$ higher than HOPG. This measurement determines the lateral resolution in CPD to about $20 \mathrm{nwn}$ with an accuracy of $5 \mathrm{mV}$ (the driving ac voltage was in this case $700 \mathrm{mV}$ ).

Of course, the influence of ambient conditions on work functions cannot be generally neglected, and we indeed observed such phenomena during our investigations. Therefore, we want 10 point out that reliable measurements of absolute work functions at ambient conditions using the currently presented method require freshly prepared surfaces of noble metals or equivalent materials as well as in sibu control of the configuration of the tip and the AFM operating mode (real noncontact mode) during data aquisition.

We have demonstrated so far that the proposed method can be used to detemine work functions quantitatively. In the following this is applied to investigate the energetics of an electrochemical reaction on WSe (p-doped single crystals with $p=10^{16} \mathrm{~cm}^{-3}$, grown with the vapor-phase transport method). ${ }^{32}$ The experiments were typically performed in the following order: At first, we determined the work function $\Phi_{\text {tip }}$ of the AFM tip by referring it to a reference sample, for which either freshly cleaved HOPG with $\Phi=-5.0 \mathrm{eV}$ or thin gold films evaporated on mica, showing (111) orientation, with $\Phi=-5.3$ $\mathrm{eV}$ were taken. With this calioration, the work function of $\mathrm{WSS}_{2}$ could then be obtained. Subsequently, on the same site of the sample, we determined the height of the dc voltage that had to be applied to induce the etching process at the surface. For improved reliability, the whole procedure was performed in one sequence within a lateral area of a few micrometers and without any changes in the setup. This implied in general that the AFM tips (cantilevers) could not be changed during an experimental session.

As already mentioned, by applying a dc voltage between an electrically conducting AFM tip and the p-doped $\mathrm{WSe}_{2}$ sample above a certain threshold, an electrochemical etching process in a physisorbed water film could be induced. ${ }^{33}$ This reaction could be further controlled and used for nanostructuring by the AFM, which is described in detail elsewhere. ${ }^{23}$ Here we want to concentrate on the energetics of the reaction. In electro- 


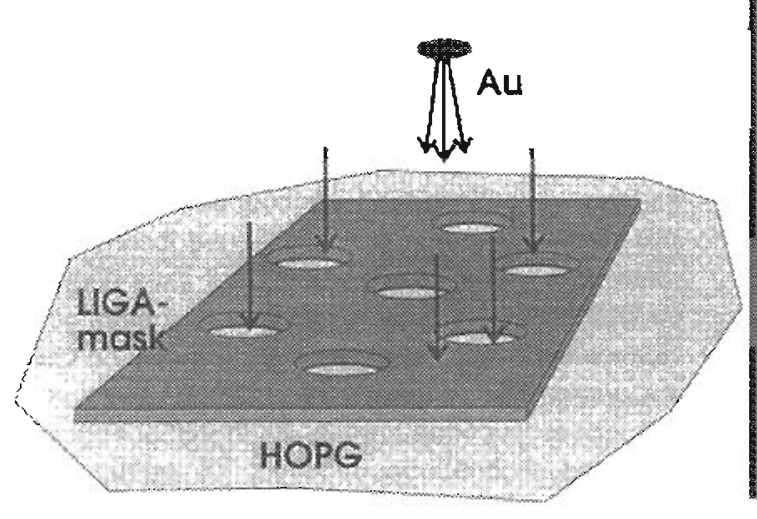

a



b

Figure 2. (a) Schematic illusustion of the patteming process of AL on HOPG. (b) Topography of the evaporated Au pattero on HOPG (AFM noncontact modc imagc; size, $30 \times 25 \mu \mathrm{m}^{2}$; gray scale, $18 \mathrm{~nm}$, black to white).

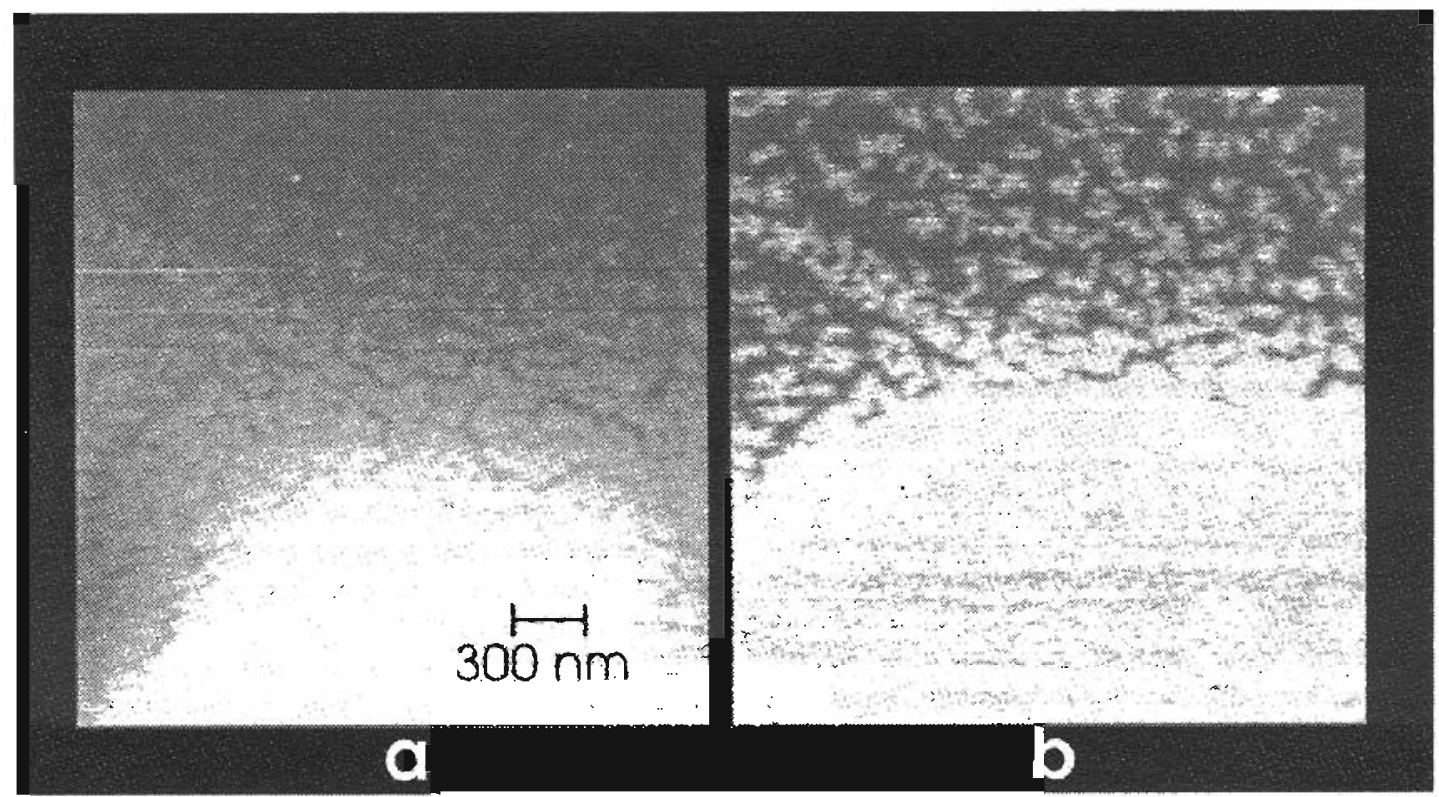

Figure 3. Gray scale images of the transition areas between Au and HOPG, simultaneously recorded with the AlFM Kelvin mode. Image size: 3 $\times 3 \mu \mathrm{m}^{2}$. (a) Topography (height difference $17 \mathrm{~nm}$ ), (b) contact poiential (image contrast: $100 \mathrm{meV}$ black to white).

chemical measurements it was found that corrosion on WSe can take place according to ${ }^{35}$

$$
\mathrm{WSe}_{2}+9 \mathrm{H}_{2} \mathrm{O}+14 \mathrm{~h}^{+} \rightarrow \mathrm{WO}_{3}+2(\mathrm{SeO} 3)^{2-}+18 \mathrm{H}^{+}
$$

In principle, this corrosion reaction can be regarded as a twostage process: First the applied dc voltage causes a bend bending in the semiconductor such that holes $\left(\mathrm{h}^{+}\right)$are driven to the surface, which then react with the physisorbed water toward nascent oxygen:

$$
\mathrm{H}_{2} \mathrm{O}+2 \mathrm{~h}^{+}-2 \mathrm{H}^{+}+\mathrm{O}
$$

In the second step, the free oxygen attacks the WSe 2 surface. The entire process will only take place if the holes are energetically able to reach the redox potential of reaction 3 , which is $+1.2 \mathrm{~V}$ against standard hydrogen electrode (SHE) or $-5.7 \mathrm{eV}$ referred to the vacuum level. The latter was derived taking the following relation between these two energy scales: $0 \mathrm{~V}$ versus $\mathrm{SHE}$ is equivalent to about $-4.5 \mathrm{eV}$ in vacuum scale. ${ }^{36}$
Without applying any external voltage, the valence band edge of the free surface is located at about -5.4 to $-5.5 \mathrm{eV}$ (refs 33,37-39), thus preventing the starting reaction. As the AFM tip and the sample are electrically connected, the Fermi level of the semiconductor is aligned to the tip's Fermi level (equal to the work function). These work functions were previously determined as described above and lie within the semiconductor's bandgap of $1.2 \mathrm{~V}$. Therefore, after approaching the tip to contact, additional band bending at the surface of $p-W e_{2}$ will be induced. This band bending varies witb the externally applied voltage until the Fermi level is shifted strong enough to reach the valence band edge (with the samplc positively biased). Then, when effects such as Fermi level pinning are neglected, the valence band edge gets shifted itself with furtber rising the sample voltage; i.e., the electron affinity is altered. Thus the energy of the holes in this simple picture is easily given by the sum of the work function of the tip and the applied dc voltage. Therefore the threshold voltage for manipulating the WSe $\mathrm{Wu}_{2}$ suface should represent the difference between the tip's work function and $-5.7 \mathrm{eV}$, which is the redox potential of reaction 3 . 
Taking these assumptions together with the work function data obtained, we were now able to predict the threshold voltages necessary for etching the surface for different tip materials. For example, one gets $(1.2 \pm 0.1)$ and $(0.3 \pm 0.1)$ $\mathrm{V}$ for the pure and the Pt-coated cantilevers, respectively. These values agree reasonably well with the experimentally determined values of $(1.5 \pm 0.1)$ and $(0.6 \pm 0.1)$ V. ${ }^{40}$

\section{Conclusion}

To summarize, we have utilized an AFM-based Kelvin probe method for investigating work functions quantitatively. This was achieved by calibrating the tip's work function to wellknown reference samples. Evidently, measuring topography and CPD simultaneously is a reliable method to obtain material contrast with high lateral resolution of $20 \mathrm{~nm}$. Additionally, we have demonstrated another major advantage of our approach in its application to the understanding of an electrochemical process. By in situ determining the Fermi level, we could derive the energetical position of the band edges of $\mathrm{p}-\mathrm{WSe}_{2}$. Using this knowledge, we were able to predict the minimum voltages necessary for initiating the electrochemical process. Moreover, CPD measurements allowed an in situ control of the configuration of the tip.

In principle, optoelectronic properties of semiconductor surfaces could also be studied by measuring the surface photovoltage induced by illumination. Especially in combination with $\mathrm{I}-\mathrm{V}$ spectroscopy, one would be able to analyze for example lateral inhomogenities of metal-semiconductor interfaces, which meets rising interest in nanoscience and technology.

Acknowledgment. We gratefully acknowledge valuable discussions with Th. Matthes and F. Mugele. We also want to thank the Institut für Mikrotechnik for providing the LIGA evaporation mask. ${ }^{30}$ This work was supported by the Deutsche Forschungsgemeinschaft (SFB 513).

\section{References and Notes} 12,515

(1) For a review, see: Wiesendanger, R. J. Vac. Sci. Technol. B 1994,

(2) Atomic and Nanometer-Scale Modification of Materials: Fundamentals and Applications; Avouris, P., Ed.; NATO ASI Series; Kluwer: Netherlands, 1993.

(3) Mizes, H. A.; Loh, K. G.; Miller, R. J. D.; Ahuja, S. K.; Grabowski, E. F. Appl. Phys. Lett. 1991, 59, 2901.

(4) Berger, C. E. H.; van der Werf, K. O.; Kooyman, R. P. H.; de Grooth, B. G.; Greve, J. Langmuir 1995, 11, 4188.

(5) Overney, R. M.; Meyer, E.; Frommer, J.; Brodbeck, D.; Lüthi, R.; Howald, L.; Güntherodt, H.-J.; Fujihira, M.; Takano, H.; Gotoh, Y. Nature 1992, 359, 133.

(6) Burger, J.; Binggeli, M.; Christoph, R.; Hintermann, H. E.; Marti, O. Forces in Scanning Probe Methods; Güntherodt, H.-J., et al., Eds.; Kluwer: Netherlands, 1995; pp 325-330.

(7) Kulik, A.; Wütherich, C.; Gremaud, G.; Briggs, G. A. D. Forces in Scanning Probe Methods; Güntherodt, H.-J., et al., Eds.; Kluwer: Amsterdam, 1995; pp 119-122.

(8) Kado, H.; Tohda, T. Jpn. J. Appl. Phys. 1997, 36, 523.

(9) Muralt, P.; Meier, H.; Pohl, D. W.; Salemink. Appl. Phys. Lett. $1987,50,1352$.

(10) Hoffmann, D.; Rettenberger, A.; Grand, J. Y.; Leiderer, P.; Dransfeld, K.; Möller, R. Thin Solid Films 1995, 264, 223.

(11) Steinke, R; Hoffmann, M.; Böhmisch, M.; Eisenmenger, J.; Dransfeld, K.; Leiderer, P. Appl. Phys. A 1997, 64, 19.
(12) Nonnenmacher, M; O'Boyle, M. P.; Wickramasinghe, H. K. Appl. Phys. Lett. 1991, 58, 2921.

(13) Itoh, J.; Nazuka, Y.; Kanemura, S.; Inoue, T.; Yokoyama, H.; Shimizu, K. J. Vac. Sci. Technol. B 1996, 14, 2105.

(14) Weaver, J. M. R.; Abraham, D. W. J. Vac. Sci. Technol. B 1991, 9, 1559 .

(15) Vatel, O.; Tanimoto, M. J. Appl. Phys. 1995, 77, 2358.

(16) Hochwitz, T.; Henning, A. K.; Levey, C.; Daghlian, C.; Slinkman, J. J. Vac. Sci. Technol. B 1996, 14, 457.

(17) Hochwitz, T.; Henning, A. K.; Levey, C.; Daghlian, C.; Slinkman, J.; Never. J.; Kaszuba, P.; Gluck, R.; Wells, R.; Pekarik, J.; Finch, R. J. Vac. Sci. Technol. B 1996, 14, 440.

(18) Weaver, J. M. R.; Wickramasinghe, H. K. J. Vac. Sci. Technol. B $1991,9,1562$.

(19) Chavez-Pirson, A.; Vatel, O.; Tanimoto, M; Ando, H.; Iwamura, H.; Kanbe, H. Appl Phys. Lett. 1995, 67, 3069.

(20) For example, see: Fillard, J. P. Near Field Optics and Nanoscopy;

World Scientific Publishing: Singapore, 1996.

(21) Lord Kelvin. Philos. Mag. 1898, 46, 82.

(22) Zisman, W. A. Rev. Sci. Instrum. 1932, 3, 367.

(23) Böhmisch, M.; Burmeister, F.; Boneberg, J.; Leiderer, P. Appl Phys. Lett. 1996, 69, 1882.

(24) Rugar, D.; Mamin, H. J.; Guethner, P. Appl Phys. Lett. 1989, 55, 2588

(25) Provided by L.O.T. Oriel GmbH, Im Tiefen See 58, D-64293 Darmstadt.

(26) Michaelson, H. B. J. Appl Phys. 1977, 11, 4729.

(27) The Si-SiO2 System; Balk, P., Ed.; Materials Science Monographs 32; Elsevier: Amsterdam, 1988.

(28) Handbook of Chemistry and Physics; Lide, D. R., Ed.; CRC Press: FL, 1995.

(29) Jägermann, W. Surface Studies of Layered Materials in Relation to Energy Converting Interfaces. In Photoelectrochemistry and Photovoltaics of Layered Semiconductors; Aruchamy, A., Ed.; Kluwer: Amsterdam, 1992; pp 195-282.

(30) The LIGA mask was kindly provided by T. Paatzsch, Institut für Mikrotechnik GmbH (IMM), Carl Zeiss Str. 18-20, D-55129 Mainz.

(31) Kern, R.; Le Lay, G.; Métois, J. J. Basic Mechanisms in the Early Stage of Epitaxy; Current Topics in Material Science 3; North-Holland: Amsterdam, 1979

(32) Al-Hilly, A.; Evans, B. J. Cryst. Growth 1972, 15, 93.

(33) Of course, the adsorption of water on $\mathrm{p}-\mathrm{WSe}_{2}$ leads to a change in work function, which had been studied in UHV by photoemission on $\mathrm{H}_{2} \mathrm{O}$ covered crystals at low temperatures. ${ }^{29,34}$ Shifts in work function of up to several $100 \mathrm{mV}$ were determined depending on the amount of water adsorbed. During our investigations we also observed a similiar change of the contact potentials with time after cleavage of the sample, which in our case was typically in the order of $150-200 \mathrm{meV}$. Additionally, in both cases the work functions shifted to lower values. Therefore we also attribute this change mainly to the adsorption of water.

(34) Mayer, T.; Jägermann, W. Synchroton Techniques in Interfacial Electrochemistry; Melendres, C. A., Tadjeddine, A., Eds.; Kluwer: Amsterdam, 1994.

(35) Lévy-Clément, C.; Tenne, R. Modification of Surface Properties of Layered Compounds by Chemical and (Photo)electrochemical Processes in Photoelectrochemistry and Photovoltaics of Layered Semiconductors; Aruchamy, A., Ed.; Kluwer: Amsterdam, 1992; pp 155-190.

(36) Morrison, S. R. Electrochemistry at Semiconductor and Oxidized Metal Electrodes; Plenum: New York, 1980.

(37) Horrocks, B. R.; Mirkin, M. V.; Bard, A. J. J. Phys. Chem. 1994, 98, 9106 .

(38) Sinn, C.; Meissner, D.; Memming, R. J. Electrochem. Soc. 1990, 137, 168.

(39) Cabrera, C. R.; Abruña, H. D. J. Electrochem. Soc. 1988, 135, 1436.

(40) However, for Pt-coated tips the situation is slightly more complicated: in an earlier paper we determined the work function of $\mathrm{Pt} / \mathrm{Ir}$ coated tips to about $-5.0 \mathrm{eV}$ and observed a threshold voltage of around $0.9 \mathrm{~V}$, which suited also well the predicted one of $0.7 \mathrm{~V} .{ }^{23}$ The reason for this discrepancy, as discovered during the present study, was an incomplete or damaged metal coverage at the very end of the tip. Therefore care was then taken to avoid such problems leading to the experimental procedure described in the former section. 